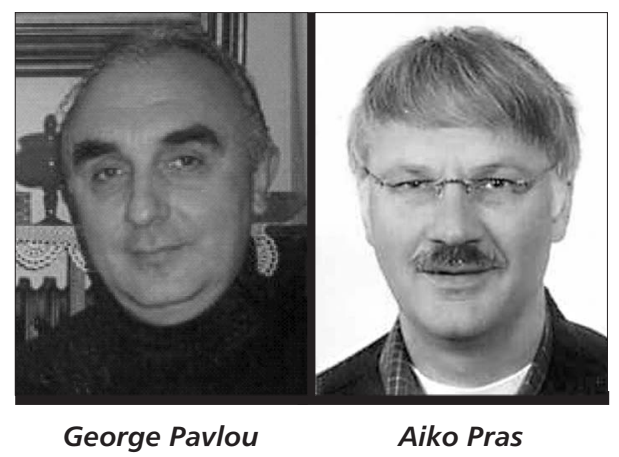

\title{
TOPICS IN NETWORK AND Service Management
}

George Pavlou

Aiko Pras

his is the sixth issue of the series on Network and

Service Management that is published twice a year, typically in April and October, although this issue has been delayed by a month. The series provides articles on the latest developments in this well established discipline, highlighting recent research achievements and providing insight into both theoretical and practical issues related to the evolution of the discipline from different perspectives. The series provides a forum for the publication of both academic and industrial research, addressing the state of the art, theory, and practice in network and service management.

An important recent development in the community was the change of chairmanship in IFIP WG6.6, the working group on the Management of Networks and Distributed Systems. Prof. Raouf Boutaba of the University of Waterloo, Canada, stepped down as chair of IFIP WG6.6 after serving two terms of three years each. Under Raouf's active leadership, the flagship management conferences Integrated Management (IM) and Network Operations and Management Symposium (NOMS) became more valuable to the community, and previously self-standing events such as DSOM, MMNS, and IPOM got integrated into Manweek. In addition, the new IEEE Transactions on Network and Service Management (TNSM) was launched. After a call for nominations, Prof. Boutaba's tasks were collectively taken over by Dr. Aiko Pras of the University of Twente, Netherlands (Chair) and Dr. Olivier Festor of INRIA, France (Vice-Chair); you may note that Dr. Pras is also Co-Editor of this series. The new chairs plan to continue the organization of successful events such as IM and NOMS, as well as smaller events such as DSOM, MMNS, and others. Key to the success of these events has been good collaboration with the IEEE Communications Society sister organisation the Committee on Network Operations and Management (CNOM). Under the leadership of the new chairs, this successful collaboration will be continued, as well as coordination and cooperation with other IFIP working groups.

Another important development for the community has been the completion of two years of integration work of the European EMANICS project on Management of the
Internet and Complex Services at the end of December 2007. This is a European Network of Excellence that brings together 13 research institutions active in the management of the future Internet. It encompasses work areas dealing with integration (long-term vision, virtual laboratory, and testbeds), dissemination (a new European conference, discussed later; electronic dissemination, training, and technology transferopen source initiatives), and joint research activities (scalable, economic, and autonomic management). For more information, visit the project site http://www.emanics.org/. You will also find there a newsletter with community news, events, and developments, which is published roughly three times a year. The network had its second yearly evaluation in March 2008, and the evaluation outcome was very positive. EMANICS has also established a European conference on Autonomous Infrastructure Security \& Management (AIMS). The second AIMS conference will take place in Bremen, Germany on 1-3 July 2008; see http://www.aims2008.org/.

The key annual event in this area, which this year was the 11th IEEE/IFIP Network Operations and Management Symposium (NOMS 2008), was held 7-11 April in Salvador, Bahia, Brazil; http://www.noms2008.org/. The second key annual event in this area is Manweek 2008, which brings together conferences and workshops such as DSOM, MMNS, and IPOM, as well as MACE, EVGM, and MGNM. This year's Manweek will take place 22-26 September, Samos Island, Greece; http://magellan.tssg.org/ 2008/.

It should finally be mentioned that Prof. George Pavlou, who is the series Co-Editor, has moved from the University of Surrey to University College London (UCL), United Kingdom, in the beginning of 2008. Prof. Pavlou was with UCL 1986-1997 as a graduate student, researcher, and lecturer, and returns to UCL after having spent 10 years as a full professor at the University of Surrey.

We again experienced an overwhelming interest in the sixth issue, receiving 32 submissions in total (two major revisions and 30 new articles), which is the highest number so far. Seven of the 30 new articles were deemed out of scope, so for the remaining 25 papers we got at least three and sometimes four independent reviews per article. We finally selected five articles, resulting in an acceptance rate 
of 20 percent. It should be mentioned that the acceptance rate for all the previous issues has ranged between 19 and 25 percent, making this series a highly competitive place to publish. We intend to maintain our rigorous review process in future issues, thus maintaining the high quality of the published articles.

The first article, "Towards an Autonomic Service Management Framework: A Holistic Vision of SOA, AON, and Autonomic Computing" by Cheng, Leon-Garcia, and Foster, attempts to consolidate efforts in service-oriented architecture, application-oriented networking, and autonomic computing. In the resulting autonomic service management framework an application with end-to-end QoS requirements can be composed from standard service components and autonomically managed according to service level agreements.

The second article, "Protocol Independent Data Modeling: Lessons Learned from the SMIng Project" by Schönwälder, discusses the author's experience from the Structure of Management Information Next Generation project that was developed within the Network Management Research Group of the Internet Research Task Force. Various aspects are discussed, including the impact of protocol style, naming independence, and exceptions, including lessons and recommendations.

The third and fourth articles both discuss management aspects of next-generation networks (NGNs). The third article, "Operators Challenges toward Bandwidth Management in DiffServ-Aware Traffic Engineering Networks" by Onali and Atzori, discusses bandwidth management problems for operators offering differentiated services who were used to simply overprovisioning network resources to fulfill user needs. It proposes an algorithm to find the adequate mapping of service types into class types and the bandwidth allocation for each of these.

The fourth article, "IMS-Controlled Ethernet Domains for Networked Services Delivery" by Tölle, Plankl, and Butscher, discusses Ethernet-based access aggregation networks that are service-controlled based on the IP Multimedia Subsystem. Quality of service support is offered, and issues related to the network attachment and resource and admission control subsystems are discussed.

Finally, the fifth article, "Fault Detection and Diagnosis in IP-based Mission Critical Industrial Process Control Networks" by Won, Choi, Hong, Kim, Hwang, Lee, and Lee, first discusses the issues of introducing IP technologies to industrial process control networks. It then proposes an architecture for fault detection and diagnosis in such networks, and proposes a failure prediction model based on operational experience with such a system.

We hope that readers of this issue again find the articles informative, and we will endeavor to continue with similar issues in the future. We would finally like to thank all the authors who submitted articles to this series, and the reviewers for their valuable feedback and comments on the articles.

\section{BIOGRAPHIES}

GEORGE PAVLOU (g.pavlou@ee.ucl.ac.uk) is a professor of communication networks in the Department of Electronic and Electrical Engineering, University College London, United Kingdom, where he coordinates the activities of the Networks and Services Research Laboratory. He received a Diploma in electrical and mechanical engineering from the National Technical University of Athens, Greece, and M.Sc. and Ph.D. degrees in computer science from University College London. His research interests focus on network management, networking, and service engineering, including aspects such as traffic engineering, quality of service management, policy-based systems, autonomic networking, multimedia service control, and object-oriented communications middleware. He has been instrumental in a number of European and U.K. research projects, and has contributed to standardization activities in ISO, ITU-T, and IETF. He was Technical Program Co-Chair of the Seventh IFIP/IEEE Integrated Management Symposium (IM 2001).

AIKo PRAS (pras@.utwente.nl) is associate professor in the Departments of Electrical Engineering and Computer Science at the University of Twente, the Netherlands, and amember of the Design and Analysis of Communication Systems Group. He received a Ph.D. degree from the same university for his thesis titled "Network Management Architectures." His research interests include network management technologies, Web services, network measurements, and accounting. He is chaior of IFIP Working Group 6.6 on Management of Networks and Distributed Systems, and is research leader of the European Network of Excellence on Management of the Internet and Complex Services (EMANICS). He has also contributed to research and standardization activities as a member of the Internet Research Task Force Network Management Research Group. He was Technical Program Co-Chair of IM 2005, and a Steering Committee member of IFIP/IEEE NOMS and IM Symposia. 